\title{
Early Detection of External Neurological Symptoms through a Wearable Smart-Glasses Prototype
}

\author{
Andrea Sciarrone, Igor Bisio, Chiara Garibotto, Fabio Lavagetto, Mehrnaz Hamedani, Valeria Prada, \\ Angelo Schenone, Federico Boero, Gianluca Gambari, Marco Cereia, and Michele Jurilli
}

\begin{abstract}
The Internet of Things (IoT) framework is moving the research community to provide smart systems and solutions aimed at revolutionizing medical sciences and healthcare. Given the extreme diffusion of Alzheimer's disease (AD) and Parkinson's disease (PD), the demand for a solution to early detect neurological symptoms of such diseases strongly arose. According to the medical literature, such early detection can be obtained through the correlation between $\mathrm{PD}$ and AD and some external symptoms: the Essential Tremor (ET) and the number of Eye Blinks (EBs). In this paper, which can be considered as an extended version of [1], we present a prototype of wearable smart glasses able to detect the presence of ET of the head and to count the number of EBs at the same time, in a transparent way with respect to the final user. Numerical results demonstrate the reliability of the proposed approach: the proposed algorithms are able to $i$ ) correctly recognize the ET with an overall accuracy above $97 \%$ and $i$ ) count the number of $E B s$ with an overall error around $9 \%$.
\end{abstract}

Index Terms-IoT, eHealth, Wearable Technology, Eye Blink, Glasses, Neurological diseases, Signal Processing.

\section{INTRODUCTION}

Alzheimer's and Parkinson's diseases (AD and PD) are some of the most well-known neurological illnesses. Namely, $\mathrm{AD}$ is the first most common neurodegenerative disease immediately followed by PD. According to the World Health Organization (WHO), in 2019 about 5.8 million people worldwide have $\mathrm{AD}$, while about 1 million people suffer from $\mathrm{PD}$ [2]. To date, some innovative therapeutic approaches for both $\mathrm{AD}$ and PD are being developed [3] [4]. Consequently, the necessity for a solution to early detect neurological symptoms of such diseases strongly arose. According to the medical literature, such early diagnosis can be achieved by exploiting the correlation between $\mathrm{PD}$ and $\mathrm{AD}$ and some external symptoms: the presence of an Essential Tremor (ET) and the counting of the number of Eye Blinks (EB). Notwithstanding

Manuscript received April 7, 2021; revised May 15, 2021. Date of publication June 9, 2021. Date of current version June 9, 2021.

A. Sciarrone, I. Bisio, C. Garibotto and F. Lavagetto are with the DITEN Department, University of Genoa, Italy, e-mails: \{name.surname $\}$ unige.it

M. Hamedani, V. Prada and A. Schenone are with the DINOGMI Department, University of Genoa, Italy, e-mails: aschenone@ neurologia.unige.it, mehrnaz.hamedani@unige.medicina.it,valeria.prada@gmail.com

F. Boero and G. Gambari are with the Gruppo FOS, Genoa, Italy, e-mails: \{name.surname\}@fos.it

M. Cereia and M. Jurilli are with FONDA Vision Health Technology, Genoa, Italy, e-mails: \{n.surname $\} @$ fondavision.com

Part of this work was presented at the 5th International Conference on Smart and Sustainable Technologies (SpliTech), 2020.

Digital Object Identifier (DOI): 10.24138/jcomss-2021-0071 the fact that $\mathrm{AD}$ and $\mathrm{PD}$ have the clearly different clinical characteristic, in some respects they have similarities. Both Parkinson's and Alzheimer's are age-related and occur later in life. They are central nervous system diseases and both of them are progressive neurodegenerative disorders which affected multiple functions. In both $\mathrm{AD}$ and $\mathrm{PD}$, the progressive damage of visual function and motor abilities can be observed [5]. Among these, the frequency of automatic blinking or/and blink reflex, could show abnormalities (either increase o decrease) in PD and to a lesser degree in AD [6]. In addition, clinical studies support the possible connections between ET and another neurodegenerative disease like PD and AD [7]. Therefore, the presence of ET may improve the risk of developing incident PD o AD during follow-up. In this paper we present NeuroGlass, a prototype of sensored-glasses already introduced in [1] and [8], able to detect the presence of ET of the head and to count the number of EBs at the same time in a transparent way with respect to the final user. NeuroGlass are equipped with 2 Inertial Measurement Units (IMUs), 1 Magnetometer and a 2-channel ElectroOculoGram (EOG). Our prototype implements some of the main state of the art aspect which are sketched in Fig. 1.

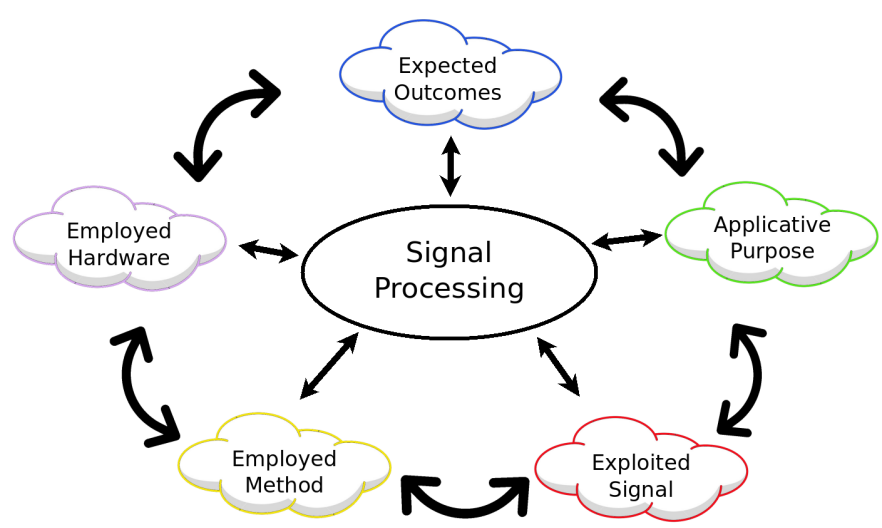

Fig. 1. Visual representation of the main aspects characterizing state-of-theart solutions

ET of the head is inferred through Short-Time-FourierAnalysis (STFA) of the inertial signals while the number of EBs is counted through a processing of the EOG signals. Even if the ET detection and the EBs counting are not innovative ideas for $\mathrm{AD}$ and/or PD diagnosis, to the best of authors' knowledge, these approaches have not been developed 
in a prototype that aims at providing a constant and daily monitoring of such symptoms at the same time. The main contributions of this work can be summarized as follows:

- we describe NeuroGlass, a new wearable and noninvasive device able to monitor neurological symptoms (ET and number of EBs) for PD and AD early detection. Our paper describes two aspects: $i$ ) the scientific approach followed and ii) the technological implementation;

- differently from the main works in the field, the proposed prototype is able to detect ET and to count the number of EBs at the same time, without the need of any a-priori training;

- in their final version, our prototype will be completely transparent with respect to the final user, who can comfortably wear it as a simple pair of glasses.

Furthermore, concerning former publications on this subject already reported as references [1] and [9], this paper provides significant additional contributions listed as follows:

- It delivers a whole brand-new medical-based Section (see Section II) which deeply discusses the main neurological motivations behind NeuroGlass idea.

- It includes a more detailed and comprehensive state of the art analysis with respect to [1], not only limited to the EB problem as in [9];

- It shows the recent advancements in the prototype evolution towards an off-the-shelf product (see Fig. 3b).

- The new numerical results reported in Figs 6, 7 and 8, which are not present in [1] and [9], have been obtained by processing data acquired through the newest version of the NeuroGlass prototype, so validating the effectiveness of the proposed final device.

Finally, obtained results prove the reliability of the proposed approach since NeuroGlass are able to: $i$ ) correctly recognize ET with an overall accuracy above $97 \%$ and $i$ ) count the number of EBs with a Root Mean Square Error (RMSE) around 0.4. The rest of this paper is organized as follows: Section III provides an overview of the state of the art. Section IV describes the NeuroGlass prototype while the algorithms for the ET detection and EBs counting are detailed in Section $\mathrm{V}$ and Section VI, respectively. Section VII presents the numerical results and, finally, conclusions are drawn.

\section{The Alzheimer's And PARKinson's Diseases}

$\mathrm{AD}$ is the most common type of dementia and it is characterized by progressive cognitive impairment [10]. Non-cognitive symptoms may be also observed like: difficulty performing motor tasks, extrapyramidal motor signs and parkinsonian symptoms including bradykinesia, gait and posture disturbances, rigidity and resting tremor [11]. It is probable that non-cognitive manifestations of $\mathrm{AD}$ are different on the basis of the location of AD pathology. The literature suggests that $\mathrm{AD}$ has a preclinical phase, during which time functional impairment may represent [11] [12]. In AD disease is also possible to observe increased blink occurrence and impaired eye-head coordination [13].
PD is characterized by the loss of nigrostriatal dopaminergic neurons. The damage of dopaminergic neurons causes impaired afferents to the striatum, leads to nerve terminal degeneration in the striatum and causes PD motor symptoms [14]. The three key elements characteristic of motor impairment in PD are bradykinesia, rigidity, and tremor [15]. Blinking is a spontaneous movement of the palpebrae due to an automatic, cyclic interplay between the elevator palpebrae superioris muscle the palpebral portion of the orbicularis oculi muscle, the reduction of blinking may be an early sign of PD [16]. Other types of movement disorders, like tremor of the eyes or of the head, can be observed in patients affected by PD sometimes early in the disease development [17].

\section{STATE OF THE ART}

a) Essential Tremor: Many current solutions are based on a combination of low cost and widely available devices such as ElectroMyoGraphy (EMG) and Intertial Measurement Units (IMUs) to monitor ET in neurological patients. The most common solution exploits the "Feature + Classifier" paradigm where features range from the peak frequency to root-meansquare, from entropy to power spectral density, from wavelet coefficients to higher order statistics [18]. Usually, these features feed classifiers such as Neural Networks (NNs), Support Vector Machines (SVMs) and Decision Trees (DTs) [18]. [19] proposes a novel method to analyze the ET of the head in patients suffering from neurological disorders by using clothes. Authors employed a set of capacitive sensors inside the collar of a shirt to detect the head position and movements. Even if the approach is non-invasive and the results are good, it cannot be extended to EBs detection. Other works monitor ET in patients through fixed medical equipment. Though such works do not provide the capability to continuously monitor the patient in his daily life, they usually produce more accurate results with respect to the ones that employ wearable devices. [20] detects the ET of the head through an analysis of EMG and accelerometric signals with the aim of predicting when tremor is about to reappear. Other interesting papers are [18], [21] that aim at differentiating ET from PD through a wearable glove by employing the "Feature + Classifier" paradigm.

b) Eye-Blink Counting: Several works aim at counting the number of EBs by observing different physical quantities. [22] exploits the Doppler signal in the joint time-frequency domain to detect eye blinking. [23] detects EBs by computing the cross correlation between the vertical and horizontal amplitude of the EOG and a template signal. [24] also exploits the EOG signal by feeding a K-Nearest Neighbour (KNN) classifier with temporal features. A very interesting work is [25], which processes the EOG signal by using the Empirical Mode Decomposition (EMD) in order to detect EBs. Similarly to NeuroGlass, [26] proposes a glasses-shaped wearable device to count the number of EBs but, differently from our proposal, it employs two cameras pointing towards the eyes. It requires a significant amount of energy to provide a full day monitoring which is a crucial aspect when it comes to wearable, and consequently portable, devices. Another very recent publication is [27] where the authors design and implement a pair of smart 
glasses (called iBlink) devised to be worn by patients suffering from facial paralysis. The main idea is to detect the EBs of the normal side of the face with a camera, through a SVM classifier, and stimulate the paralyzed side so that the blinks of both eyes becomes symmetric. Finally, in [28] an overall graphical representation of some of the main works of the state of the art is sketched in Figure 2.

\section{The NeuroGlass Prototype}

\section{A. Wearability Specifications}

From the mere aesthetic viewpoint NeuroGlass is shaped like a traditional pair of glasses, equipped with normal lenses, designed to be comfortably worn by anyone who needs glasses. However, in designing the NeuroGlass frame, we had to take into account several aspects that are of primary importance due to the nature of the device. First, the frame has been designed with the goal of making the device usable also for people that needs to wear non conventional lenses (e.g. prismatic lenses or lenses with a high power). In particular we relied on a round design flattened on top, in order to support the wider set of ophtalmic lenses; this choice has the advantage of making the device wearable to a wide class of patients, without the need of producing several frame with different designs. Second, the frame of NeuroGlass is not a simple spectacle frame used only to support ophthalmic lenses, but it has also at least other two key functions:

- its structure hosts electrodes and must ensure a reliable contact between these elements and the face/head of the patient.

- it must be able to enclose the electronic board and components for data acquisition and transmission as well as a storage unit and power source.

In order to address the first point, some frame parts, namely the nosepads and the tample tips, are conceived to allow the adjustement of the position of the frame with respect the face of the patient. Thanks to this solution, it is possible to give the patient the best comfort experience and at the same is possible to adjust the contact points of the electrodes placed on the frame. In addition, such solution allows NeuroGlass to be worn firmly so avoiding unwanted glasses movements that could produce to noisy acquisitions leading to false alarms, missed detection and wrong outcomes.

For how concerning the second point, designing a frame with spaces for electronic components is not a trivial task, because the process must take into account the trade off between the increase of the dimensions of the frame elements (dictated by the size of the electronic boards to embed) and usability and comfort needs. The solution we propose adds to the frame a housing in each temple and an additional housing in the front part. Housings have been appositely created to embed the electronic boards and components in the frame, and are hidden by removable covers, in order to allow an easy access to the processing logic, for maintenance operations.

Fig. 3 shows the electronic skeleton of the proposed prototype (left part) and its latest version (right part).

\section{B. Hardware Specifications}

The final version of NeuroGlass compared to the prototype has a more ergonomic and wearable shape, which implies less available space for the hardware. This has led to the need to review the spaces for the various components and their arrangement on the PCB. Compared to the first version, in which the components were placed on the entire frame of the glasses, the second one has the circuitry only on one of the two temples. In the prototype the components on the frame were arranged as follows: the microchip (ADS1299) and its circuitry dedicated to the acquisition of the EOG signals were placed on the right temple, on the left side were placed the NRF52832 microcontroller, the SD allocation and the IMUs, eventually other IMUs components were positioned of the front side of the frame. In the final version of the glasses all the previous are installed only on the left temple, while the other one is prepared for the allocation of the battery source. Due to the lack of space some of the components were replaced whit a single item, like the IMUs and the magnetometer (LSM9DS1). Finally, our prototype has a 2channel EOG in order to the detect and count the number of EBs. The device acquires data from the two IMUs, the magnetometer and the EOG channels with a sampling rate $f_{s}$ equal to $100 \mathrm{~Hz}$. Data can be transmitted to a desktop PC via a USB interface using a simple comma-separated format or they can be stored in a SD card inserted in a slot mounted on the left temple for later download. Moreover, the final version has a button to control the acquisition, a LED for the feedback of the acquisition, some hardware components for communication via BLE. Eventually the firmware has been optimized using OS FreeRTOS to increase and synchronize the sample frequency between IMUs and EOG sensors.

\section{Essential Tremor (ET) Detection}

Head ET can be divided into two categories: Nodding tremor (similar to a nodding movement) and Negation tremor (similar to a negation movement). Differently from most of the existing literature, our approach does not require any training to detect the presence of the tremor and distinguish its typology. The employed algorithm is divided into two steps:

1) Decide if ET is present by comparing the overall Instantaneous Frequency (IF) $\psi$ with a threshold $\zeta_{\psi}$;

2) if step 1) detects the ET, discriminate its typology by computing the energies of different signals.

We define $\mathbf{s}^{p}, \mathbf{s} \in \boldsymbol{\Sigma}=\{\mathbf{a}, \mathbf{g}, \mathbf{m}\}$ and $p \in \boldsymbol{\Omega}=\{T M P, B R G\}$ the general three-component vector containing the accelerometer/gyroscope/magnetometer samples acquired by the IMU placed on the glass temple $(T M P)$ or bridge $(B R G)$. For example, $\mathbf{a}^{T M P}, \mathbf{m}^{T M P}$ represent the three-component accelerometer and magnetometer vectors, respectively, sensed by the IMU on the glasses temple (see the superscript $T M P$ ). The quantity $s_{i}^{p}(n)$ is the $n$-th sample of the $i$-th component, belonging to the generic $s$ signal sensed by the IMU in $p$ position where $n \in[1, N]$ and $i \in\{x, y, z\}$. For example, $a_{x}^{T M P}(4)$ is the fourth sample of $x$-component of the accelerometer vector sensed by the IMU on the temple. 


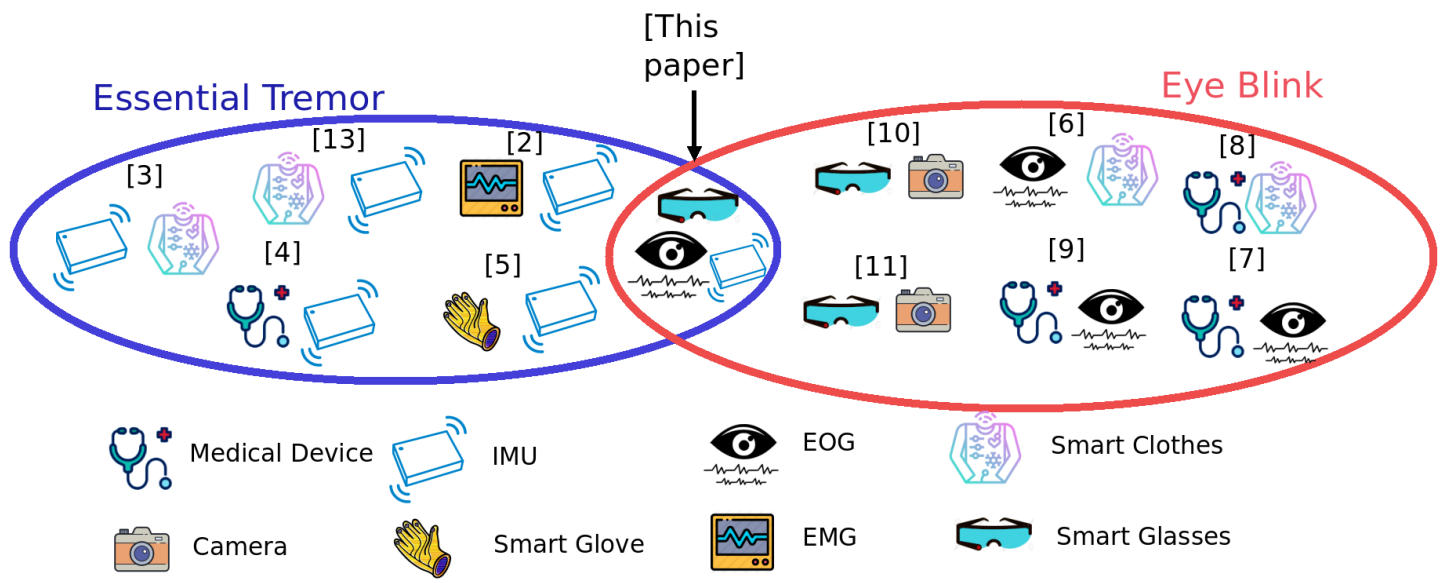

Fig. 2. Graphical representation of some of the main works of the state of the art.

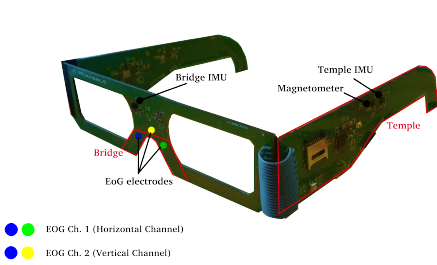

(a) Prototype's electronics

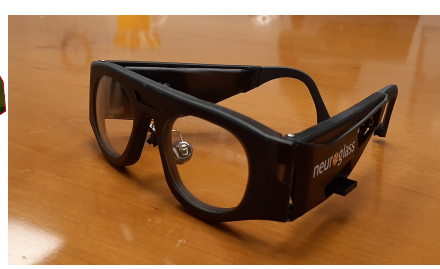

(b) Final prototype
Fig. 3. The temporal evolution of the proposed NeuroGlass prototype. Fig. 3a shows the prototype's electronics along with an illustration of the employed sensors and of the main glasses components while Fig. $3 \mathrm{~b}$ shows the prototype in its final version.

The first step is carried out by a standard pre-processing stage composed of a mean removal, Low Pass Filter (LPF) with cutoff frequency of $5 \mathrm{~Hz}$ and a Hamming windowing, thus obtaining the pre-processed signal $\tilde{\mathbf{s}}_{i}^{p}$. Then, $\tilde{\mathbf{s}}_{i}^{p}$ is divided into $N$ frames, each of them containing $N_{f}$ samples. In this paper $N_{f}=200$ and each frame is overlapped with the next one by an overlap percentage of $50 \%$.

We define $\mathbf{S}_{i}^{p}=\mathcal{F}\left[\tilde{\mathbf{s}}_{i}^{p}\right]$ the discrete spectrogram obtained from the generic pre-processed signal $\tilde{\mathbf{s}}$, related to the $i$-th axis and placed in position $p$, through the Short-Time Fourier Analysis $\mathcal{F}$. Consequently, $S_{i}^{p}(k, n)$ is the single complex value of the spectrogram $\mathbf{S}_{i}^{p}$ for the frequency index $k$ and the time index $n$ with $n \in[1, N]$ and $k \in\left[0, N_{f}-1\right]$.

Considering that ET has prominent frequency components, from the quantity $S_{i}^{p}(k, n)$ the estimation of the Instantaneous Frequency (IF) is performed. The rationale behind this idea is simple: if tremor is present, then the spectral analysis will coherently provide a high-energy IF estimation. According to the literature, several methods exist to accomplish such a task. An effective solution consists in computing the weighted average of all frequencies occurring in the spectrogram [29]. Another possibility, which requires some hypothesis on the noise, is to approximate the IF with the frequency that maximizes the energy of each time slice of the spectrogram. Finally, another idea leverages the employment of the Hilbert transform but it requires a signal in the time-domain and it usually fails when the signal is not strictly mono-component. In this paper the IF $\tilde{f}_{s, i}^{p}(n)$ related to the signal $s$, for the $i$-th axes in the $p$ position, relative to the $n$-th time index is estimated as follows:

$$
\tilde{f}_{s, i}^{p}(n)=\frac{f_{s}}{2 N_{f}} \cdot \frac{\sum_{k=0}^{N_{f}-1} k \cdot\left|S_{i}^{p}(k, n)\right|^{2}}{\sum_{k=0}^{N_{f}-1}\left|S_{i}^{p}(k, n)\right|^{2}}, \quad \forall n \in[1, N]
$$

From the single IFs $\tilde{\mathbf{f}}_{s, i}^{p}$ (i.e., related to specific signals and axes) an overall IF $\psi$ can be computed. In order to provide such computation the percentage of energy with respect to the total energy related to the estimated IFs $\tilde{f}_{s, i}^{p}(n)$ must be determined. Such values $e_{s, i}^{p}(n) \in[0,1]$ can be computed as follows:

$$
e_{s, i}^{p}(n)=\frac{\left|S_{i}^{p}(\tilde{k}(n), n)\right|^{2}}{\max _{k \in\left[0, N_{f}-1\right]}\left|S_{i}^{p}(k, n)\right|^{2}}, \quad \forall n \in[1, N]
$$

where

$$
\tilde{k}(n)=\tilde{k}_{s, i}^{p}(n)=\left\lfloor\frac{2 N_{f}}{f_{s}} \cdot \tilde{f}_{s, i}^{p}(n)\right\rfloor, \quad \forall n \in[1, N]
$$

is the nearest discrete frequency index associated to the estimated IFs $\tilde{f}_{s, i}^{p}(n)$ and the symbol $\lfloor\cdot\urcorner$ is the rounding operator. The overall IF $\psi$ can be obtained by using the following formula:

$$
\psi=\sum_{p \in \boldsymbol{\Omega}} \sum_{s \in \boldsymbol{\Sigma}} \sum_{i \in\{x, y, z\}} \tilde{f}_{s, i}^{p} \cdot e_{s, i}^{p}
$$

where $\tilde{f}_{s, i}^{p}=\frac{1}{N} \sum_{n=1}^{N} \tilde{f}_{s, i}^{p}(n)$ is the mean value of the single IFs for the $i$-th axis, the $s$-th signal sensed by the IMU in the $p$ position.

The ET is detected by comparing the overall IF $\psi$ with an empirically pre-determined threshold $\zeta_{\psi}$ : if $\psi \geq \zeta_{\psi}$ then the presence of ET has been detected. No tremor is found if $\psi<\zeta_{\psi}$. In this paper the value $\zeta_{\psi}=2 \mathrm{~Hz}$ has been 
set. The rationale behind the approach and the choice of $\zeta_{\psi}$ relies on the fact that former studies (see [21] and references therein) have pointed out that the ET presents frequency components with significant energies that range from 3 to 5 $\mathrm{Hz}$. Conversely, if no tremor is present, the spectral energy distribution has some peaks around the DC component while it tends to flatten for all the other frequencies. The overall IF contains such information: a low value of $\psi$ states that there is no tremor while a large value indicates the presence of the tremor. The classification between Nodding tremor and Negation tremor can be achieved considering the energy along specific axes of the wearable device. Taking into account the physical movements of the head during these two types of tremor ("up\&down" for the Nodding tremor and "left\&right" for the Negation one), we can distinguish them by exploiting the amount of energy of the following signals: $\mathbf{g}_{x}^{B R G}, \mathbf{g}_{y}^{B R G}$, $\mathbf{a}_{x}^{B R G}, \mathbf{a}_{y}^{B R G}$. If the energy $\phi_{N G T}$ present in $\mathbf{g}_{x}^{B R G}$ and $\mathbf{a}_{x}^{B R G}$ is greater than the energy $\phi_{N D D}$ present in $\mathbf{g}_{y}^{B R G}$ and $\mathbf{a}_{y}^{B R G}$ then the tremor will be classified as Negation tremor. In the opposite case, it will be classified as Nodding tremor. The overall amount of energy $\phi_{N G T}$ and $\phi_{N D D}$ can be obtained as follows:

$$
\begin{aligned}
& \phi_{N G T}=\sum_{s \in \mathbf{\Sigma}^{*}} \sum_{i \in \mathbf{\Lambda}^{N G T}} w_{s, i}^{B R G} \cdot e_{s, i}^{B R G} \\
& \phi_{N D D}=\sum_{s \in \boldsymbol{\Sigma}^{*}} \sum_{i \in \mathbf{\Lambda}^{N D D}} w_{s, i}^{B R G} \cdot e_{s, i}^{B R G}
\end{aligned}
$$

where $\boldsymbol{\Sigma}^{*} \subset \boldsymbol{\Sigma}=\{\mathbf{g}, \mathbf{a}\}, \boldsymbol{\Lambda}^{N G T}=\{x\}$ and $\boldsymbol{\Lambda}^{N D D}=\{y\}^{1}$. The values $w_{s, i}^{B R G}$ are weights employed to enhance one axis with respect to another. They have been computed $a$-priori as:

$$
w_{s, i}^{p}=\frac{\sum_{n=1}^{N} e_{s, i}^{p}(n)}{\sum_{s \in \mathbf{\Sigma}} \sum_{i \in\{x, y, z\}} \sum_{n=1}^{N} e_{s, i}^{p}(n)}, \quad \forall p \in\{T M P, B R G\}
$$

The whole ET algorithm is summarized in the pseudo-code reported in the Algorithm box 1.

\section{Eye BLINK (EB) COUNTING}

The detection of the number of EBs is performed through the processing of the EOG signal, which is an inexpensive and non-invasive way of recording eye ball movements. The source for the EOG signal is cornea-retinal potential generated due to the movements of eye balls within the conductive environment of the skull [30]. In the literature some of the most employed algorithms for detecting the number of blinks range from the "features + classifier" paradigm [23], [24] to wavelet transforms [30], from dynamic time warping [31] to signal decomposition [25].

In this paper we propose a novel pre-processing stage for the EOG signal denoising, followed by a matched filter applied to

\footnotetext{
${ }^{1}$ The employment of the sets $\boldsymbol{\Lambda}^{N G T}$ and $\boldsymbol{\Lambda}^{N D D}$, both composed of single element, allow a more general description of the problem since different axes could be employed to estimate the energies $\phi_{N G T}$ and $\phi_{N D D}$.
}

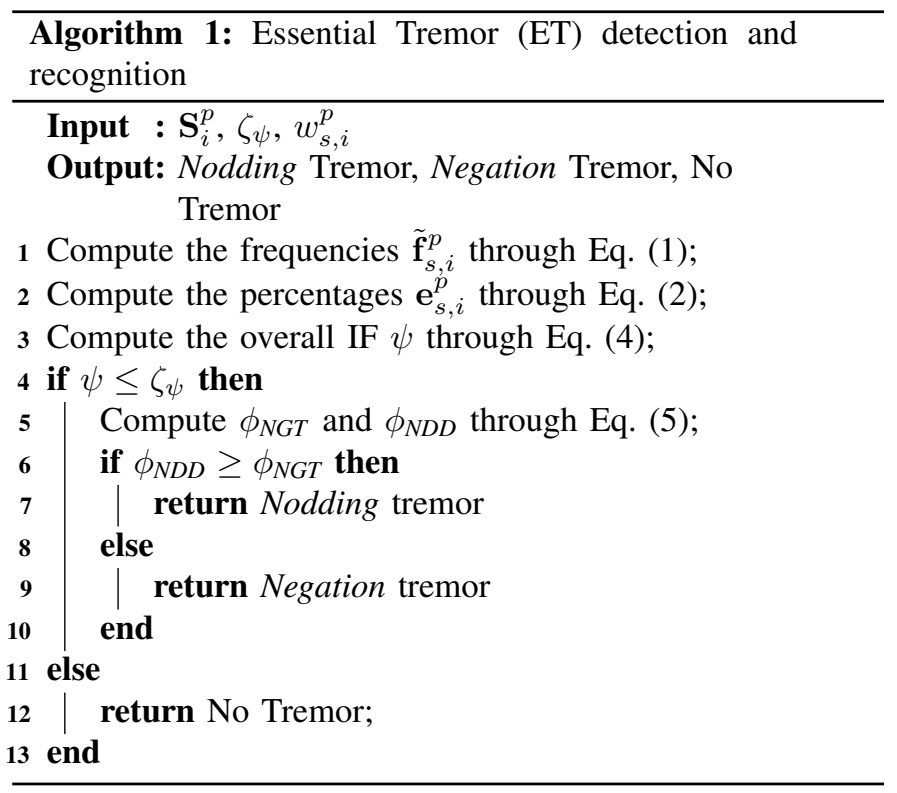

the second-order derivative. The first step consists setting two thresholds $\epsilon_{1}$ and $\eta_{\text {ref }}$ : this is done by initializing $\epsilon_{1}=10^{24}$ and $\eta_{\text {ref }}=0.95$. Then, the mean is removed from $s(n)$, thus obtaining a new signal $s^{\prime}(n)$. The threshold $\epsilon_{2}=\alpha \cdot \sigma\left[s^{\prime}(n)\right]$ is computed, where $\sigma$ is the standard deviation and $\alpha$ is a smoothing factor set to 0.5 that trades the severity of the threshold operation. $s^{\prime}(n)$ is then filtered through a non-linear, soft-threshold block to obtain the signal $s^{\prime \prime}(n)$ as follows:

$$
s^{\prime \prime}(n)= \begin{cases}\frac{1}{2 T} \sum_{t=1}^{T} s^{\prime}(n-t)+s^{\prime}(n+t), & \text { if }\left|s^{\prime}(n) \geq \epsilon_{2}\right| \\ s^{\prime}(n)-\epsilon_{2}, & \text { if }\left|s^{\prime}(n)<\epsilon_{2}\right|\end{cases}
$$

where $T$ is the filter span, set to 5 in this paper. Finally, the ratio $\eta=\frac{\epsilon_{2}}{\epsilon_{1}}$ is computed and compared with its reference threshold $\eta_{\text {ref }}$ : if $\eta \leq \eta_{\text {ref }}$ then $\epsilon_{1}$ is updated with the values of $\epsilon_{2}$ (i.e., $\epsilon_{1}=\epsilon_{2}$ ). Vice versa if $\eta>\eta_{\text {ref }}$ the pre-process stage concludes by applying a LPF with cutting frequency set to $3[\mathrm{~Hz}]$, thus obtaining from $s^{\prime \prime}(n)$ the preprocessed signal $\tilde{s}(n)$. The EBs detection algorithm employs a matched filter to the second-order derivative of the signal $\tilde{s}(n)$. The reason for the second-order derivative is two-fold: i) it allows enhancing the abrupt changes in the signal and ii) it provides a more identifiable waveform for the matched filter $m(n)$. To build such matched filter we have recorded EOG acquisitions of about $30 \mathrm{~s}$ from 5 people, sampling at $f_{s}=100[\mathrm{~Hz}]$. Among these acquisitions we have manually isolated $5 \mathrm{EBs}$ for each subject so obtaining $B=25$ different blinks. From these blinks the vector $\Theta$ is built so that $\boldsymbol{\Theta}=\left[\tilde{s}_{1}(n), \tilde{s}_{2}(n), \cdots \tilde{s}_{b}(n), \cdots \tilde{s}_{B}(n)\right]$, where $\tilde{s}_{b}(n)$, $n \in[0, Q-1]$ is a pre-processed EOG sequence containing just one blink and $b \in[1, B]$ is the blinking index. On each element of the vector $\Theta$ the second-order discrete derivative is computed and the result is finally averaged, thus obtaining the sequence $\bar{s}(n)$ as follows: 


$$
\bar{s}(n)=\frac{1}{B} \sum_{b=1}^{B} \Delta^{2}\left[\tilde{s}_{b}(n)\right]
$$

where $\Delta^{2}$ denotes the second-order discrete derivative. Finally, we define the matched filter $m(n)=\bar{s}(Q-1-n), \forall n \in$ $[0, Q-1]$ where $Q$ is the length of the sequence $\bar{s}(n)$.

To count the number of EBs from a new EOG acquisition $s(n)$, the pre-processing stage is applied so as to obtain the filtered signal $\tilde{s}(n)$. Then, $\tilde{s}(n)$ is divided in frames where each frame is denoted with $\tilde{s}_{l}(n), l \in\left[0, N_{f}-1\right]$. Each frame is then convoluted with the matched filter $m(n)$ and its maximum value is extracted to obtain a new quantity $\lambda(l)$ defined as follows:

$$
\lambda(l)=\max _{n \in[0, \Xi-1]}\left[\sum_{\xi} \tilde{s}_{l}(\xi) m(n-\xi)\right], \quad \forall l \in\left[0, N_{f}-1\right]
$$

where $\Xi$ is the length of the convolution between $\tilde{s}_{l}$ and $m$. The number of EBs $\rho$ is determined as in Eq.10:

$$
\rho=\sum_{l=0}^{N_{f}-1} \Psi[\lambda(l), \beta \cdot \bar{\lambda}]
$$

where $\bar{\lambda}=\frac{1}{N_{f}} \sum_{l=0}^{N_{f}-1} \lambda(l)$ is the average value of $\lambda(l)$ and $\Psi[\lambda(l), \beta \cdot \bar{\lambda}]=1$ if $\lambda(l) \geq \beta \cdot \bar{\lambda}$ and 0 otherwise. $\beta$ is a simple constant set to 1.5 .

\section{NUMERICAL RESULTS}

This section reports the obtained numerical results, both for the ET detection and for the counting of the number of EB. Since this is a preliminary work, we have tested our device on normal subjects that simulated ET and provided EBs. Concerning the ET, from 5 people 15 different acquisitions have been taken: 5 with no tremor, 5 with Nodding tremor and 5 with Negation tremor. Each acquisition lasts for about 30 seconds.

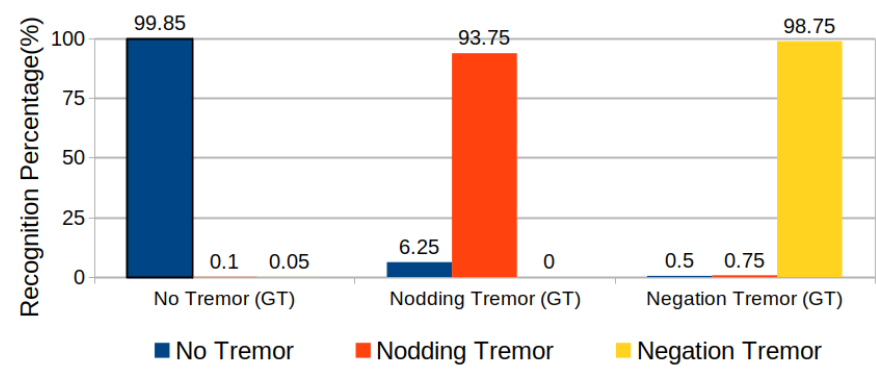

Fig. 4. Bar plot of the tremor detection accuracy. The overall accuracy is $97.45 \%$.

The obtained results are reported in Fig. 4. The No Tremor case is almost perfectly recognized with a correct decision percentage above 99\%. Similarly, the Nodding tremor and the Negation tremor are detected with a correct decision percentage of $93.75 \%$ and $98.75 \%$, respectively.
The $6.25 \%$ of the cases wrongly classified as No Tremor belong to acquisitions where the tremor had lower energy. Summarizing, the provided algorithm is able to recognize the ET and to determine its typology with an overall accuracy of above $97 \%$.

To validate the proposed EBs detection algorithm, we have sensed through our prototype 5 different EOG acquisitions from 5 people for an overall dataset of 25 EOG acquisitions. In each acquisition the number of EBs ranges from 8 to 24 and each acquisition lasts for about 30 seconds.

To obtain the best possible signal quality the electrodes must be placed in specific positions on the patient's face. Unfortunately, this constraint cannot be granted by a wearable IoT device that trades the electrodes' position with the comfort of wearing simple glasses. In other words, the shape of the glasses forces specific positions for the electrodes which are different from the optimal ones. This great dissimilarity in hardware setup leads to the need of a different approach for EOG signal processing. In order to better understand the impact of the EOG electrodes positioning, we have considered two different configurations for the EOG signal acquisition:

- Optimal: it is the same electrodes positioning related to professional medical desktop equipment. It is the best electrodes positioning.

- Glasses: the electrodes position follows the physical contact points of the glasses with the face.

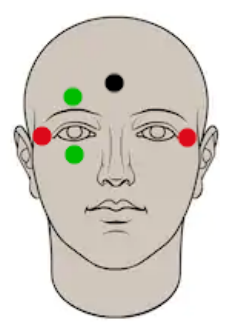

Optimal

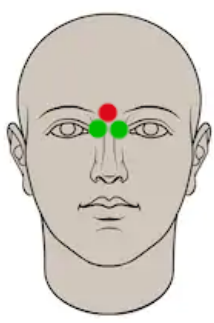

Glasses

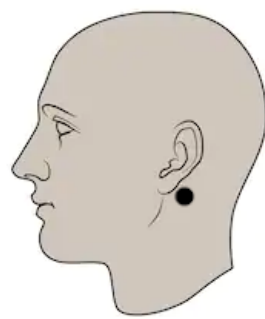

Fig. 5. The two configurations used in experimental tests for the position of EOG electrodes: optimal configuration (left) and glasses configuration (right).

Figure 5 shows a graphical representation of both the configurations used in the experimental tests where the red dots represent the horizontal channel, the green dots refer to the vertical one and the black dots correspond to ground.

In the optimal configuration, the electrodes are put above and beneath one eye, and at the external corner of both eyes, while ground is measured on the forehead. This configuration is shown in Figure 5 (left part). In the literature, many papers acquire the EOG signal by using this configuration [25]. Although this configuration achieves minimal perturbation on the measured signals, it is a very uncomfortable setup for the patient due to the intrusiveness of the solution. Moreover, the electrodes position in this approach cannot be granted by using wearable glasses as EOG sensing device since the electrodes must be put where the glasses frame has contact with the patient's skin. These specific locations are the glasses bridge, located at the top of the nose, and the temples, where reference 
TABLE I

Root Mean SQUare ERror (RMSE) AS A FUNCTION OF THE FRAME LENGTH (IN SECONDS) AND OF THE OVERLAP PERCENTAGE.

\begin{tabular}{ccc}
\hline $\begin{array}{c}\text { Frame } \\
\text { length [s] }\end{array}$ & Overlap (\%) & RMSE \\
\hline \hline \multirow{3}{*}{0.5} & 0.4 & 0.63 \\
& 0.6 & 0.66 \\
& 0.8 & 0.53 \\
& 1 & 0.84 \\
\hline \hline & 0.4 & 1.12 \\
0.7 & 0.6 & 1.42 \\
& 0.8 & 1.39 \\
& 1 & 1.47 \\
\hline \hline & 0.4 & 2.25 \\
1 & 0.6 & 2.19 \\
& 0.8 & 2.42 \\
& 1 & 2.49 \\
\hline \hline
\end{tabular}

ground is measured. This configuration is shown in Figure 5 (right part). Furthermore, the optimal configuration allows the patient to wear our prototype firmly so avoiding unwanted glasses movements that could produce to noisy signals.

Since the optimal configuration provides the best results in terms of performance, in the present work we run experimental tests by using the optimal EOG configuration as a benchmark for the glasses one.

Table I reports the Root Mean Square Error (RMSE) as a function of the frame length (in seconds) and of the overlap percentage. The global minimum point is reached with a frame length of 0.53 seconds with an overlap equal to $75 \%$, where the RMSE is around 0.44 .

For this specific case other plots, reported in the next figures see Figs. 6, 7 and 8, provide some more details regarding the actual number of EBs (blue bars) versus the estimated number of EBs $\rho$ (orange bars) for each acquisition, so highlighting the accuracy of the proposed algorithm.

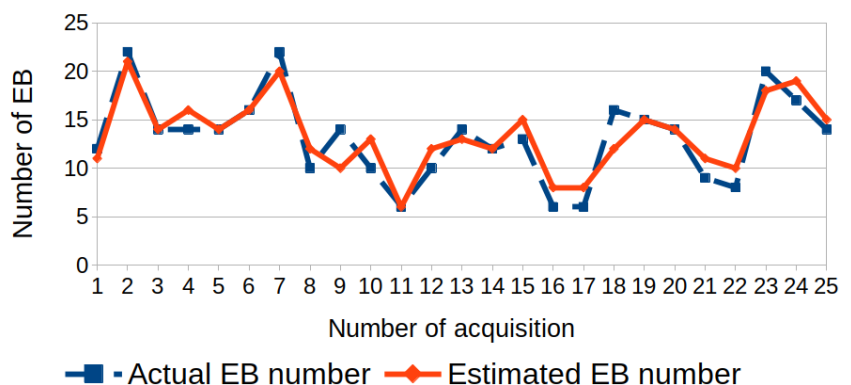

Fig. 6. Plot of the actual number of EBs (dashed blue line) versus the estimated number of EBs $\rho$ (continuous orange line) for each acquisition. This result is related to the electrodes in the "Glasses" position.

\section{CONCLUSIONS}

This paper presents NeuroGlass, a prototype of sensored glasses able to detect the presence of Essential Tremor (ET) of the head and to count the number of Eye Blink (EB) at the same time without any a-priori training. Numerical results prove the reliability of the proposed approach: our algorithms are able to $i)$ correctly recognize the ET with

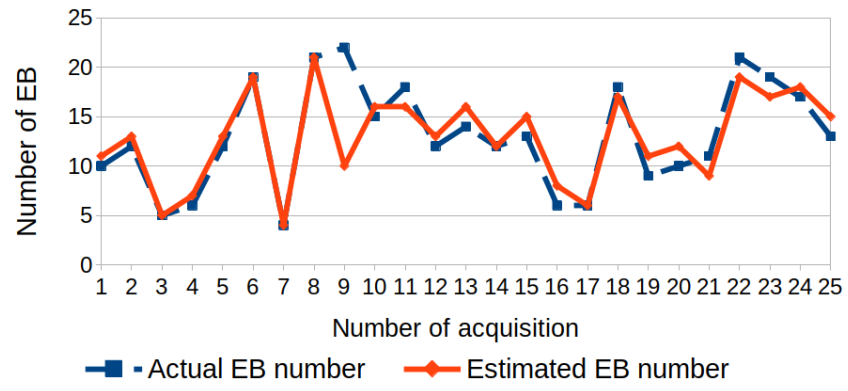

Fig. 7. Plot of the actual number of EBs (dashed blue line) versus the estimated number of EBs $\rho$ (continuous orange line) for each acquisition. This result is related to the electrodes in the "Optimal" position.

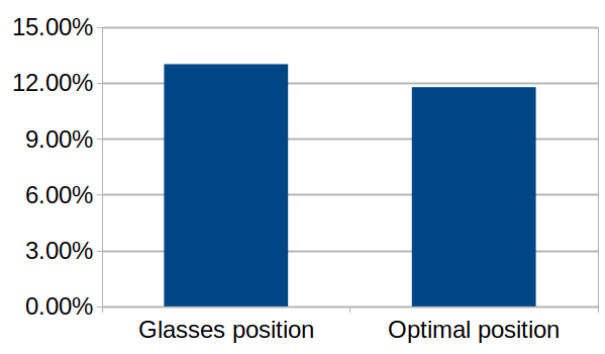

Fig. 8. Plot of the actual number of EBs (blue bars) versus the estimated number of EBs $\rho$ (orange bars) for each acquisition.

an overall accuracy above $97 \%$ and ii) count the number of EBs with a Root Mean Square Error (RMSE) around 0.44. Finally, considering that our solution both for the cases of ET and $\mathrm{EB}$ detection does not require any a-priori training nor heavy calculations, its computational cost is low. However, a study aimed at estimating the computational complexity of the proposed algorithm is currently ongoing and it will be published in a future extension of this manuscript.

\section{ACKNOWLEDGMENT}

This research has been partially founded by the NeuroGlass project within the "P.O.R. FESR LIGURIA 2014-2020" call, Axis 1 "Research and Innovation", Action 1.2.4.

\section{REFERENCES}

[1] A. Sciarrone, I. Bisio, C. Garibotto, F. Lavagetto, G. Staude, and A. Knopp, "A wearable prototype for neurological symptoms recognition," in ICC 2020 - 2020 IEEE International Conference on Communications (ICC), 2020, pp. 1-7.

[2] D. S. Woodruff-Pak, M. Papka, S. Romano, and Y.-T. Li, "Eyeblink classical conditioning in alzheimer's disease and cerebrovascular dementia,' Neurobiology of Aging, vol. 17, no. 4, pp. 505 - 512, 1996.

[3] D. Colombo, P. Pnevmatikou, E. Melloni, and C. Keywood, "Therapeutic innovation in Parkinson's disease: a 2020 update on disease-modifying approaches," Expert Review of Neurotherapeutics, vol. 20, no. 10, pp. 1047-1064, Oct. 2020. [Online]. Available: https://www.tandfonline.com/doi/full/10.1080/14737175.2020.1800454

[4] L. Piemontese, "An innovative approach for the treatment of Alzheimer's disease: the role of peroxisome proliferator-activated receptors and their ligands in development of alternative therapeutic interventions," Neural Regeneration Research, vol. 14, no. 1, p. 43, 2019. [Online]. Available: http://www.nrronline.org/text.asp?2019/14/1/43/241043 
[5] A. Bargagli, E. Fontanelli, D. Zanca, I. Castelli, F. Rosini, S. Maddii, I. Di Donato, A. Carluccio, C. Battisti, G. M. Tosi, M. T. Dotti, and A. Rufa, "Neurophthalmologic and Orthoptic Ambulatory Assessments Reveal Ocular and Visual Changes in Patients With Early Alzheimer and Parkinson's Disease," Frontiers in Neurology, vol. 11, p. 577362, Nov. 2020. [Online]. Available: https://www.frontiersin.org/articles/10.3389/fneur.2020.577362/full

[6] J. S. Leon-Ariza, D. G. Prada, D. S. Leon-Ariza, C. Castillo, and F. E. Leon-Sarmiento, "The Three Nociceptive Responses of the Orbicularis Oculi Reflex in Alzheimer's Disease: State of the Evidence and Meta-analysis," Clinical EEG and Neuroscience, vol. 50, no. 5, pp. 354-360, Sep. 2019. [Online]. Available: http://journals.sagepub.com/doi/10.1177/1550059418825169

[7] H. LaRoia and E. D. Louis, "Association between Essential Tremor and Other Neurodegenerative Diseases: What Is the Epidemiological Evidence?" Neuroepidemiology, vol. 37, no. 1, pp. 1-10, 2011. [Online]. Available: https://www.karger.com/Article/FullText/328866

[8] A. Sciarrone, I. Bisio, C. Garibotto, F. Lavagetto, M. Hamedani, V. Prada, S. Stara, A. Schenone, F. Boero, and G. Gambari, "Neuroglasses: A wearable prototype for early detection of neurological symptoms," in 2020 5th International Conference on Smart and Sustainable Technologies (SpliTech), 2020, pp. 1-6.

[9] A. Sciarrone, I. Bisio, C. Garibotto, F. Lavagetto, G. H. Staude, and A. Knopp, "Leveraging iot wearable technology towards early diagnosis of neurological diseases," IEEE Journal on Selected Areas in Communications, vol. 39, no. 2, pp. 582-592, 2021.

[10] A. Calderone, M. Formenti, F. Aprea, M. Papa, L. Alberghina, A. M Colangelo, and P. Bertolazzi, "Comparing Alzheimer's and Parkinson's diseases networks using graph communities structure," BMC Systems Biology, vol. 10, no. 1, p. 25, Dec. 2016. [Online]. Available: https://bmcsystbiol.biomedcentral.com/articles/10.1186/s12918016-0270-7

[11] A. S. Buchman and D. A. Bennett, "Loss of motor function in preclinical Alzheimer's disease," Expert Review of Neurotherapeutics, vol. 11, no. 5, pp. 665-676, May 2011. [Online]. Available: http://www.tandfonline.com/doi/full/10.1586/ern.11.57

[12] A. S. Buchman, R. S. Wilson, P. A. Boyle, J. L. Bienias, and D. A Bennett, "Grip Strength and the Risk of Incident Alzheimer's Disease," Neuroepidemiology, vol. 29, no. 1-2, pp. 66-73, 2007. [Online]. Available: https://www.karger.com/Article/FullText/109498

[13] D. B. Carr and P. Grover "The Role of Eye Tracking Technology in Assessing Older Driver Safety," Geriatrics, vol. 5, no. 2, p. 36, Jun. 2020. [Online]. Available: https://www.mdpi.com/2308-3417/5/2/36

[14] E. M. Prasad and S.-Y. Hung, "Behavioral Tests in Neurotoxin-Induced Animal Models of Parkinson's Disease," Antioxidants, vol. 9, no. 10, p. 1007, Oct. 2020. [Online]. Available: https://www.mdpi.com/2076$3921 / 9 / 10 / 1007$

[15] J. Jankovic, "Parkinson's disease: clinical features an diagnosis," Journal of Neurology, Neurosurgery \& Psychiatry, vol. 79, no. 4, pp. 368-376, Apr. 2008. [Online]. Available: https://jnnp.bmj.com/lookup/doi/10.1136/jnnp.2007.131045

[16] C. Simonet, A. Schrag, A. J. Lees, and A. J. Noyce, "The motor prodromes of parkinson's disease: from bedside observation to large-scale application,” Journal of Neurology, Dec. 2019. [Online]. Available: http://link.springer.com/10.1007/s00415-019-09642-0

[17] R. J. Leigh and S. Martinez-Conde, "Tremor of the eyes, or of the head, in Parkinson's disease?" Movement Disorders, vol. 28, no. 6, pp. 691-693, Jun. 2013. [Online]. Available: https://onlinelibrary.wiley.com/doi/10.1002/mds.25478

[18] C. Thanawattano, C. Anan, R. Pongthornseri, S. Dumnin, and R. Bhidayasiri, "Temporal fluctuation analysis of tremor signal in parkinson's disease and essential tremor subjects," in 2015 37th Annual International Conference of the IEEE Engineering in Medicine and Biology Society (EMBC), Aug 2015, pp. 6054-6057.

[19] M. Hăgan, A. Constantinescu, and O. Geman, "Tremor analysis in neurological disorders using intelligent clothes," in 2015 E-Health and Bioengineering Conference (EHB), Nov 2015, pp. 1-4

[20] P. Shukla, I. Basu, and D. Tuninetti, “Towards closed-loop deep brain stimulation: Decision tree-based essential tremor patient's state classifier and tremor reappearance predictor," in IEEE Engineering in Medicine and Biology Society, Aug 2014, pp. 2605-2608.

[21] P. Locatelli and D. Alimonti, "Differentiating essential tremor and parkinson's disease using a wearable sensor - a pilot study," in 2017 7 th IEEE International Workshop on Advances in Sensors and Interfaces (IWASI), June 2017, pp. 213-218.
[22] Y. Kim, "Detection of eye blinking using doppler sensor with principal component analysis," IEEE Antennas and Wireless Propagation Letters, vol. 14, pp. 123-126, 2015.

[23] M. Nakanishi, Y. Mitsukura, Y. Wang, Y.-T. Wang, and T. Jung, "Online voluntary eye blink detection using electrooculogram," 2014.

[24] M. Vidal, A. Bulling, and H. Gellersen, "Analysing eog signal features for the discrimination of eye movements with wearable devices," in Proceedings of the 1st International Workshop on Pervasive Eye Tracking \&\#38; Mobile Eye-based Interaction, ser. PETMEI '11. New York, NY, USA: ACM, 2011, pp. 15-20. [Online]. Available: http://doi.acm.org/10.1145/2029956.2029962

[25] M. S. Reddy, A. Sammaiah, B. Narsimha, and K. S. Rao, "Analysis of eog signals using empirical mode decomposition for eye blink detection," in 2011 International Conference on Multimedia and Signal Processing, vol. 2, May 2011, pp. 293-297.

[26] H. Le, T. Dang, and F. Liu, "Eye blink detection for smart glasses," in 2013 IEEE International Symposium on Multimedia, Dec, pp. 305-308.

[27] X. Tian, X. Zheng, Y. Ji, B. Jiang, T. Wang, S. Xiong, and X. Wang, "iBlink: A wearable device facilitating facial paralysis patients to blink," IEEE Trans. on Mobile Computing, vol. 18, no. 8, pp. 1789-1801, 2019.

[28] N. M. Kumar, N. Kumar Singh, and V. K. Peddiny, "Wearable smart glass: Features, applications, current progress and challenges," in 2018 Second International Conference on Green Computing and Internet of Things (ICGCIoT), Aug 2018, pp. 577-582.

[29] R. Romulus, "A comparison between instantaneous frequency estimation methods of frequency modulated signals covered with gaussian noise," in 2012 10th International Symposium on Electronics and Telecommunications, Nov 2012, pp. 331-334.

[30] M. S. Reddy, B. Narasimha, E. Suresh, and K. S. Rao, "Analysis of eog signals using wavelet transform for detecting eye blinks," in 2010 International Conference on Wireless Communications Signal Processing (WCSP), Oct 2010, pp. 1-4.

[31] M. Rusydi, T. Okamoto, S. Ito, and M. Sasaki, "Rotation matrix to operate a robot manipulator for $2 \mathrm{~d}$ analog tracking objects using electrooculography," Robotics, vol. 3, pp. 289-309, 092014

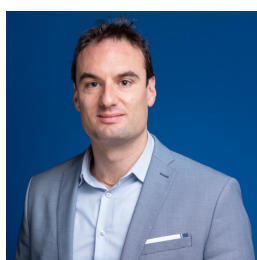

Andrea Sciarrone was born in Livorno, Italy in 1984. He got his Master Degree Cum Laude in Telecommunication Engineering in 2009 in the same university. In 2014 he got the $\mathrm{Ph}$. D. degree in Ambient Intelligence at University of Genoa where he is currently Assistant Professor and member of the DSP Laboratory. His research concerns Signal Processing over Internet of Things, Context and Location Awareness and Safety and e-health Applications. Currently, he is Vice-Chair of the IEEE ComSoc eHealth Technical Committee.

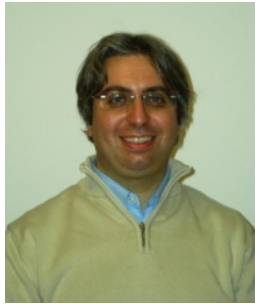

Igor Bisio got his "Laurea" degree in 2002 and his Ph.D. degree in 2006, both at the University of Genoa, Italy. He is currently Associate Professor and member of the research staff of the Telecommunication Research Group and, in particular, of the Digital Signal Processing (DSP) Laboratory at the University of Genoa. He is IEEE Communications Society (ComSoc) Member. His research concerns IoT Signal Processing, Context and Location Awareness, Adaptive Coding, Safety and e-health Applications, Satellite Communication systems.

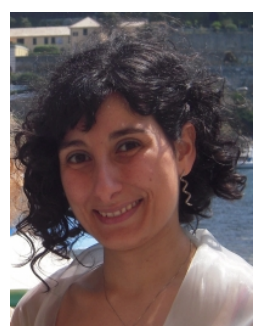

Chiara Garibotto was born in Chiavari, Italy in 1985. In 2015 she achieved a Master Degree in Multimedia Signal Processing and Telecommunication Networks and in 2019 she got a PhD degree in Science and Technology for Electronic and Telecommunication Engineering. Currently she is Research Fellow at the DSPLab at the University of Genoa. Her main research activities concern Multimedia Signal Processing, Context Awareness for Smart Environments, Safety \& Security and e-Health applications. 


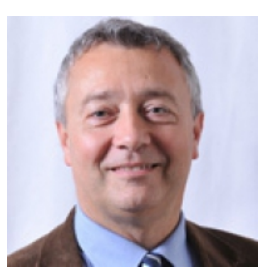

Fabio Lavagetto is full professor in Telecommunications at the University of Genoa and since 2016 is member of the Board of Directors in the same university. Currently he is the head of research of the Digital Signal Processing (DSP) Laboratory of the University of Genoa and he has authored over 100 scientific publications in international journals and conferences. His current research concerns Signal Processing over Internet of Things, Context and Location Awareness, Safety \& eHealth applications and satellite communication systems.

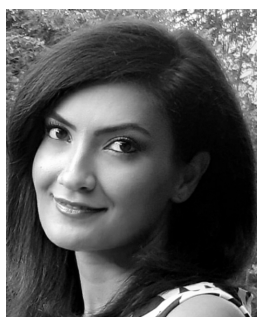

Mehrnaz Hamedani is a physiotherapist, she achieved a Ph.D. in Neuroscience from the University of Genoa. Currently, she is working as a postdoctoral fellow at the Department of Neuroscience, Rehabilitation, Ophthalmology, Genetics, Materna and Child Health, IRCCS AOU San Martino-IST (DINOGMI) at the University of Genoa. Her main research activities focused on the development of specific sensors to monitor movements during rehabilitative protocols.

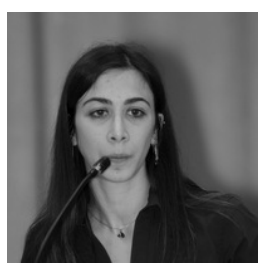

Valeria Prada achieved a Bachelor of Science Degree in Biology and a Master Science in Biology at the University of Milan. She obtained the Ph.D. in Neuroscience at University of Genoa and the Degree of Physiotherapy at the same University. Currently she is working as a postdoctoral fellow at the Department of Neuroscience, Rehabilitation, Ophthalmology, Genetics, Maternal and Child Health, IRCCS AOU San Martino-IST (DINOGMI) at the University of Genoa.

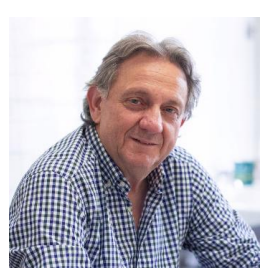

Angelo Schenone is full professor at University of Genoa and Director of Neurology Unit of San Martino Polyclinic Hospital. He is the Coordinator of the Bachelor Degree in Physiotherapy and the $\mathrm{PhD}$ in Neuroscience program. Prof. Schenone is also the coordinator of the Master Degree in Telerehabilitation assisted by technologies. Since 2016 he is president of Italian Society of the Periphera Nervous System and since 2015 he is member of the Board of the Italian Society of Neurology. Finally, he is the ordinary partner of Peripheral Nerve Society; Italian Society of Neurology and Italian society of Neuropathology. He is author of 250 scientific publications in national/international journals and over 500 press releases at national and international conferences.

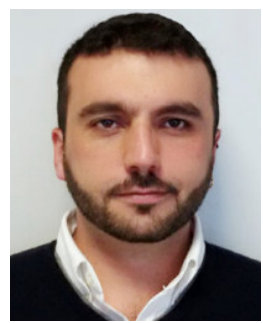

Federico Boero received the B.Sc. and M.Sc. degrees in electronic engineering from the University of Genoa, Genoa, Italy, in 2007 and 2010, respectively. Since 2010, he has been a Project Engineer with the Department of Research and Development in Gruppo FOS, Genoa with experience in analogical and digital circuit and system design for sensing and automation. His current research interests include Internet of Things, RF systems, industrial and biomedical applications.

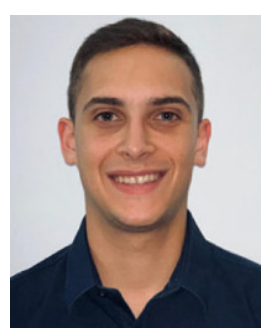

Gianluca Gambari was born in Genoa, in 1995. He received the master's degree in data science and engineering from the University of Genoa, in 2020. He has been currently working as a Project Engineer with Research and Development for about two years in Gruppo Fos.

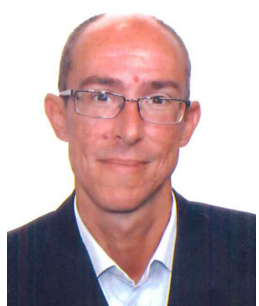

Marco Cereia received the Laurea Degree in electronic engineering from the Politecnico di Torino in 2001. From 2001 to 2014 he was a Researcher with the National Research Council of Italy (CNR), in the field of industrial communication networks. Since 2014 Marco Cereia has been with FONDA srl company, where he deals with several technical aspects related to smart medical devices for visually impaired people, including research and development in the field of smart glasses.

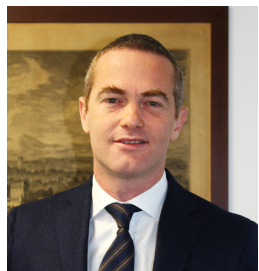

Michele Jurilli is an entrepreneur in the field of medical devices starting form 2010 in the family company Optics International. Michele funded FONDA Vision Health Technology company in the year 2012, succeeding in the development of business and patented technologies, both in vision rehabilitation and wearable technology. 